\title{
The role of dietary fibre in inflammatory bowel disease
}

\author{
Aleksandra Pituch-Zdanowska, Aleksandra Banaszkiewicz, Piotr Albrecht \\ Department of Paediatric Gastroenterology and Nutrition, Medical University of Warsaw, Warsaw, Poland
}

Prz Gastroenterol 2015; 10 (3): 135-141

DOI: $10.5114 /$ pg.2015.52753

Key words: Crohn's disease, ulcerative colitis, dietary fibre.

Address for correspondence: Aleksandra Pituch-Zdanowska, Department of Paediatric Gastroenterology and Nutrition, Medical University of Warsaw, 1 Dzialdowska St, 01-184 Warsaw, Poland, phone: +48 224523 310, fax: +48 2245 23 310, e-mail: aleksandra.pituch@wum.edu.pl

\begin{abstract}
The aetiology of inflammatory bowel diseases (IBD), which are primarily Crohn's disease and ulcerative colitis, still remains unclear, while the incidence of IBD is constantly increasing, especially in the industrialised countries. Among genetic, environmental, and immunological factors, changes in the composition of the intestinal microflora and diet are indicated as very important in initiating and sustaining inflammation in patients with IBD. Above all nutrients dietary fibre is an especially important component of diet in the context of IBD. A potentially protective effect of high-fibre diet on intestinal disorders was described as early as in 1973. Several trials performed in animal models of IBD and human studies have reported that supplementation of some types of dietary fibre can prolong remission and reduce lesions of the intestinal mucosa during the course of the disease. This paper presents the current state of knowledge on the effects of dietary fibre in IBD.
\end{abstract}

\section{Introduction}

Despite the progress in medicine, the aetiology of inflammatory bowel diseases (IBD), which are primarily Crohn's disease (CD) and ulcerative colitis (UC), still remains unclear. What is more, the incidence of IBD, especially $C D$, is still increasing and the highest rates are found in the industrialised countries. It is worrying that in recent years the age of onset is still decreasing and a significant increase in morbidity has been observed among children and adolescents. In the paediatric population the diagnosis is made mainly between 5 and 16 years of age, although IBD occurs more often also among toddlers and infants [1, 2].

It is believed that the disease process develops as a result of interactions of genetic, environmental, and immunological factors. Also, changes in the composition of the intestinal microflora are crucial in the pathogenesis of IBD [3-5]. Diet is a major factor affecting enteric flora, and several studies have considered the role of specific nutrients in the development of IBD and in the course of the disease. The so-called Western diet, which is characterised by high dietary intake of monosaccharides and disaccharides and low intake of dietary fibre, has been linked with increased risk of both CD and UC in many studies [6].
Dietary fibre is an especially important component of diet in the context of IBD. A potentially protective effect of a high-fibre diet on intestinal disorders was pointed out by Burkitt in 1973 [7] when he worked in African countries and observed a low incidence of colon cancer and other non-infectious intestinal diseases among the inhabitants, whose diet was normally rich in dietary fibre. However, it is commonly observed that patients with IBD are permanently on a low-fibre diet, regardless of disease activity $[8,9]$. The European Crohn's and Colitis Organisation emphasised that continuation of a regular diet is recommended during mildly to moderately active disease, both in UC and CD patients. The fibre-restricted diet should always be used only on a temporary basis, and it is indicated in a few cases, which are acute relapse (with diarrhoea, cramping), intestinal stenosis, and small intestinal bacterial overgrowth and after some types of surgery. Clinical practice guidelines summarising dietary advice regarding fibre intake in IBD (taking into account disease activity) are shown in Table I [10].

It is the intention of the authors of this paper to review available data about the potential anti-inflammatory role of dietary fibre in the course of IBD. 
Table I. Clinical practice guidelines summarising dietary advice regarding fibre intake in IBD. Based on [10]

\begin{tabular}{|c|c|}
\hline Phases/states of the IBD & Guidelines \\
\hline $\begin{array}{l}\text { Moderate/severe disease activity, fistulas, strictures, } \\
\text { after surgery (e.g. colectomy) }\end{array}$ & $\begin{array}{l}\text { Low-fibre diet, especially without: } \\
\text { - whole-grain products, brown rice, wild rice, bran } \\
\text { - corn, corn hulls, popcorn } \\
\text { - nuts, seeds } \\
\text { - raw fruits, fruits with seeds (except peeled apple, ripe banana and melon) } \\
\text { - beans } \\
\text { - raw vegetables, potatoes with skins } \\
\text { - prune juice }\end{array}$ \\
\hline Periods of no symptoms, no or mild disease activity & $\begin{array}{l}\text { Slow reintroduction of high-fibre foods one at a time in small amounts, } \\
\text { to meet } \mathrm{Al}^{*} \text { recommendations for dietary fibre }\end{array}$ \\
\hline Ulcerative proctitis & Consider high-fibre diet in case of constipation \\
\hline
\end{tabular}

${ }^{*}$ Al - Adequate intake for age and gender.

\section{Dietary fibre - a definition}

According to the American Association of Cereal Chemists (AACC), dietary fibre is "the edible parts of plants or analogous carbohydrates that are resistant to digestion and absorption in the human small intestine, with complete or partial fermentation in the large intestine". In compliance with this definition dietary fibre includes non-starch polysaccharides and resistant oligosaccharides (e.g. cellulose, hemicelluloses, pectin), analogous carbohydrates (e.g. resistant potato dextrins), lignin, and substances associated with the non-starch polysaccharide and lignin complex in plants (e.g. waxes, cutin) [11]. The Codex Alimentarius Commission (CAC) definition from 2009 is that "dietary fibre means carbohydrate polymers with three or more monomeric units, which are neither digested nor absorbed in the small intestine of humans" and this is the main, current, operative definition ofdietary fibre around the world [12]. It is worth mentioning that there are many other, considered definitions, and lately Jones [13] made a concentrated review of the literature on this topic.

Of the main types of dietary fibre, the most important, from a physiological point of view, are water soluble

Table II. Substrates for the production of each SCFA (\% of total SCFA production) [16]

\begin{tabular}{lccc} 
Substrate & Butyrate & Acetate & Propionate \\
\hline Resistant starch & 38 & 41 & 21 \\
\hline Oat bran & 23 & 57 & 21 \\
\hline Wheat bran & 19 & 57 & 15 \\
\hline Cellulose & 19 & 61 & 20 \\
\hline Guar gum & 11 & 59 & 26 \\
\hline Pectin & 9 & 75 & 14
\end{tabular}

(e.g. fructans, pectin, $\beta$-glucan) and water insoluble (e.g. cellulose, some hemicelluloses, lignin) dietary fibres. Although in 1998 the experts of the Food and Agriculture Organisation of the United Nations/World Health Organisation (FAO/WHO) proposed to withdraw this division due to the fact that the solubility is not always associated with a particular physiological effect [14], this division is still used and current. Dietary fibre can be then divided into fractions that are rapidly fermented (e.g. oligosaccharides), slowly fermented (e.g. gums), and those that are hardly fermented, like wheat bran.

Soluble fibre is a type of fibre that dissolves in water. It attracts the water to form a viscous gel and it tends to slow the colonic transit time. Almost all of the soluble fibre fractions are completely fermented in the large bowel. Non-fermentable water-insoluble fibres increase the volume of stool and by mechanical stimulation/irritation of gut mucosa decrease faecal transit time. Fermentable insoluble fractions, such as some forms of resistant starch (RS), undergo partial fermentation by the gut microbiota. Resistant starch is the sum of starch and starch degradation products that are not digested and absorbed in the small intestine and get unaltered into the colon [15]. Resistant starch has been categorised into four types (RS1-4). It can bring some of the benefits of water-insoluble fibre and some of the benefits of water-soluble fibre.

During bacterial fermentation of non-digestible dietary fibreshort chain fatty acids (SCFA), mainly acetate, propionate, and butyrate, and gases: carbon dioxide, hydrogen, and methane, are produced. Butyrate is considered to be the main energy substrate for colonocytes and a factor that stimulates their growth and differentiation. The RS is regarded as the most butyrogenic (Table II) [16]. In general SCFA are considered to be crucial in maintaining intestinal homeostasis, and the best-known mechanism of their action is the inhibition of proinflammatory mediator activities in the intestinal epithelium [17]. 
Some examples of dietary fibre content in food are shown in Table III [18]. As well as natural sources of fibre there are widely available fibre supplements that are considered to be a form of a subgroup of function- al dietary fibre. According to the Institute of Medicine (IOM), functional fibre "consists of isolated, non-digestible carbohydrates that have beneficial physiological effects in humans", and it may be isolated or extracted

Table III. Examples of dietary fibre content in food (g/amount) [18]

\begin{tabular}{|c|c|c|c|c|}
\hline Content & Amount (weight) & Total fibre $[\mathrm{g}]$ & Insoluble fibre [g] & Soluble fibre [g] \\
\hline \multicolumn{5}{|l|}{ Fruits: } \\
\hline Apple, fresh with skin & 1 medium (138 g) & 3.73 & 2.76 & 0.97 \\
\hline Banana, fresh or ripe & 1 medium (118 g) & 2.83 & 2.12 & 0.71 \\
\hline Cranberries, dried & $1 \mathrm{C}(120 \mathrm{~g})$ & 6.01 & 4.44 & 1.56 \\
\hline Kiwi & 1 medium (76 g) & 2.58 & 1.98 & 0.61 \\
\hline Mango, fresh & 1 each (207 g) & 3.73 & 2.19 & 1.53 \\
\hline Orange, fresh & 1 medium (131 g) & 3.14 & 1.31 & 1.83 \\
\hline Strawberries, fresh & $1 \mathrm{C}(144 \mathrm{~g})$ & 3.31 & 2.45 & 0.86 \\
\hline \multicolumn{5}{|l|}{ Vegetables: } \\
\hline Asparagus, cooked & $1 \mathrm{C}(180 \mathrm{~g})$ & 2.88 & 1.44 & 1.44 \\
\hline Broccoli, cooked from fresh & 1 C (156 g) & 4.68 & 2.34 & 2.34 \\
\hline Carrot, raw & 1 C (110 g) & 3.30 & 1.65 & 1.65 \\
\hline Carrot, cooked from fresh & $1 \mathrm{C}(156 \mathrm{~g})$ & 5.15 & 2.96 & 2.18 \\
\hline Cauliflower, cooked from fresh & $1 \mathrm{C}(125 \mathrm{~g})$ & 3.37 & 2.50 & 0.87 \\
\hline Corn, cooked & 1 medium (100 g) & 2.80 & 2.30 & 0.50 \\
\hline Lentils, cooked from dried & 1 C (198 g) & 15.64 & 14.45 & 1.19 \\
\hline Potato, boiled without skin & $1 \mathrm{C}(156 \mathrm{~g})$ & 2.81 & 1.20 & 1.61 \\
\hline Sauerkraut & $1 C(236 \mathrm{~g})$ & 5.90 & 3.85 & 2.05 \\
\hline \multicolumn{5}{|l|}{ Grains: } \\
\hline Bread, multigrain & 1 slice (26 g) & 1.79 & 1.48 & 0.31 \\
\hline Bread, pumpernickel & 1 slice $(26 \mathrm{~g})$ & 1.51 & 0.73 & 0.78 \\
\hline Millet, cooked & $1 \mathrm{C}(240 \mathrm{~g})$ & 6.48 & 5.26 & 1.22 \\
\hline Oat bran & 1 C (219 g) & 5.67 & 3.02 & 2.65 \\
\hline Oatmeal, regular cooking & $1 \mathrm{C}(234 \mathrm{~g})$ & 3.98 & 2.13 & 1.85 \\
\hline Rice brown, cooked & 1 C (195 g) & 3.51 & 3.28 & 0.23 \\
\hline Wheat bran & $1 \mathrm{C}(64 \mathrm{~g})$ & 27.39 & 25.22 & 2.18 \\
\hline \multicolumn{5}{|l|}{ Others: } \\
\hline Almonds & 1 C (142 g) & 15.90 & 14.34 & 1.56 \\
\hline Flax seeds & 1 C (114 g) & 25.46 & 11.57 & 13.98 \\
\hline Dark chocolate & $1 \mathrm{oz}(28.35 \mathrm{~g})$ & 1.67 & 1.60 & 0.07 \\
\hline Sunflower seeds & 1 C (128 g) & 13.44 & 10.75 & 2.69 \\
\hline Potato chip & $1 \mathrm{oz}(28.35 \mathrm{~g})$ & 1.31 & 0.56 & 0.75 \\
\hline
\end{tabular}


using chemical, enzymatic, or aqueous steps [19]. Fibre supplements are available in powders, tablets, and capsules. The most common sources of fibre in available supplements are natural, such as psyllium, glucomannan, inulin, wheat dextrin, or flax, and synthetic, such as methylcellulose and calcium polycarbophil.

\section{Dietary fibre in animal models of inflammatory bowel diseases}

So far, many studies in experimental animal models of IBD have been conducted, evaluating the anti-inflammatory effect of different fractions of fibre. In most studies the therapeutic effect has been linked with an increased luminal production of SCFA (especially butyrate) after the administration of appropriate sources of dietary fibre. These studies are summarised in the next sections.

In the trinitrobenzenesulfonic acid (TNBS) model of rat colitis, administering a fibre-supplemented diet (5\% Plantago ovata seeds) for two weeks prior to TNBS-colitis induction, and thereafter for 1 week, contributed to a significant reduction of intestinal inflammation. The anti-inflammatory effect was associated with lower tumour necrosis factor $\alpha$ (TNF- $\alpha$ ) levels and lower nitric oxide synthase (NOS) activity in the large intestine as compared with non-treated colitic rats. Furthermore, it was observed that the study group had higher concentrations of the SCFA (mainly butyrate and propionate) in the intestinal contents [20]. In HLA-B27 transgenic rats, administering a diet enriched with Plantago ovata seeds for 13 weeks resulted in a significant decrease in some of the pro-inflammatory mediators, such as NOS, leukotriene B4, and TNF- $\alpha$. As in previous studies, fibre-treated colitic rats had higher concentrations of butyrate and propionate in the intestinal contents than the control group [21].

Oral inulin supplementation (1\% in drinking water, or $400 \mathrm{mg} /$ day) reduced the severity of dextran sodium sulphate (DSS) colitis in rats, as evidenced by significantly lower lesion scores, decreased release of mediators, and lower tissue myeloperoxidase (MPO) activity compared with a control group. Oral inulin also lowered colonic $\mathrm{pH}(\mathrm{pH}<7.0)$ and increased counts of lactobacilli [22]. In HLA-B27 transgenic rats the anti-inflammatory effect of oral supplementation of oligofructose-enriched inulin (OF-IN) was associated with alterations to the gut microbiota (significant increases in caecal Lactobacillus and Bifidobacterium), decreased tissue proinflammatory cytokines, and increased immunomodulatory molecules [23]. Short chain fructo-oligosaccharides (SC-FOS) supplementation in the TNBS model of colitis resulted in a decrease of MPO activity, lower leukotriene B4 production, and lower NOS expression. The anti-inflam- matory effect was also evaluated macroscopically, and a significant reduction was observed in the extent of colonic damage. Also SC-FOS affected the composition of intestinal microflora by increasing Lactobacilli and Bifidobacteria counts [24]. Short-chain inulin-like fructans with degree 4 of polymerisation (DP4) have accelerated the healing process of TNBS-induced colitis in rats. The DP4 supplementation resulted in a decrease in MPO activity and an increase in Lactobacilli and Bifidobacteria counts. In addition, there was an increase in faecal SCFA concentrations, propionate in particular [25]. Supplementation of fructo-oligosaccharides (FOS) in HLA-B27 transgenic rats significantly reduced colitis and increased Bifidobacteria in all FOS-fed rats. Whereas only in $50 \%$ of inulin-fed rats was intestinal inflammation decreased. In the faecal samples of both groups a decrease of total bacteria and Clostridium cluster XI and decrease of gene copies of Clostridium difficile toxin $B$ were observed. The changes were positively correlated with a reduction of chronic intestinal inflammation [26].

In DSS-induced colitis model, supplementation of RS resulted in a significant improvement of caecal and distal colonic mucosa, assessed macroscopically and histologically. The healing effect was associated with significantly higher amounts of butyrate in the cecum of the RS-DSS rats [27]. The RS is regarded as butyrogenic in particular.

Kanauchi et al. [28] and Araki et al. [29] showed that oral supplementation of germinated barley foodstuff (GBF), which is rich in glutamine and low-lignified hemicelluloses, in DSS-induced colitis in rats prevented mucosal damage and increased mucosal protein and RNA contents compared to the control group. It was also shown that GBF had an inhibitory effect on mucosal mast cells, thereby preventing lesions of the intestinal submucosa.

\section{Dietary fibre studies in humans}

In 1978 Davies and Rhodes [30] performed a study evaluating the impact of high-fibre diet on maintaining clinical remission in patients with UC. Patients in remission were divided into two groups. The first group $(n=15)$ continued on sulfasalazine without any changes in diet while the second group $(n=24)$ increased dietary fibre intake by including whole-wheat bread, vegetables, and $25 \mathrm{~g}$ of wheat bran per day. The diet was well tolerated by the majority of patients, so they discontinued sulfasalazine after 2 weeks of the study. Only 4 patients were unable to tolerate the high-fibre diet, and they were excluded from the study. Control visits were determined after 1, 3, and 6 months from the start of the intervention. Relapses were observed in $20 \%$ of patients on sulfasalazine and in $75 \%$ of patients 
on the high-fibre diet. The conclusion of the authors was that the high-fibre diet did not maintain remission in patients with UC. No beneficial effect was associated with the type of fibre used. Whole-wheat food is mainly composed of water-insoluble and non-fermentable fibre. Furthermore, it appears that dietary fibre can only be one of the elements in UC therapy and cannot replace the use of appropriate drugs.

Hallert et al. [31], in their placebo-controlled trial, showed that supplementation of Plantago ovata husk may relieve gastrointestinal symptoms in patients with UC in remission. After 4 months of intervention, most of the patients from study group (69\%) reported alleviation of symptoms compared with a placebo-control group (24\%). The differences were statistically significant $(p<0.001)$. In another clinical trial, the effect of Plantago ovata seeds on maintaining clinical remission in patients with UC was assessed. A total of 105 patients in remission were randomly assigned to one of three groups to receive Plantago ovata seeds (10 g twice daily), mesalamine (0.5 g three times a day), and Plantago ovata seeds plus mesalamine at the same doses as the previous groups. The study included $102 \mathrm{pa}$ tients. After 12 months, failure in maintenance of remission was observed in $40 \%$ of patients from the Plantago ovata seeds group, 35\% in the mesalamine group, and $30 \%$ in the Plantago ovata plus mesalamine group. The results demonstrated the similar effectiveness of all therapies in maintaining remission in patients with UC and the good tolerance of the dietary fibre used. In addition, increased butyrate concentrations in the stools of patients from Plantago ovata seeds group were observed $(p=0.018)$ [32].

In a randomised, double-blind, crossover design study, 20 patients with asymptomatic pouchitis received $24 \mathrm{~g}$ per day of inulin or placebo for 3 weeks. After the intervention it was observed that the inulin supplementation resulted in a reduction of disease activity both in endoscopic and histological assessment (pouchitis disease activity index - PDAI). Inulin increased butyrate concentrations, lowered colonic $\mathrm{pH}$, and decreased the number of Bacteroides fragilis and concentrations of secondary bile acids in faeces [33]. In another randomised, placebo-controlled trial 67 patients with inactive and mild to moderately active $C D$ were randomised to receive oligofructose-enriched inulin (OF-IN) or placebo $10 \mathrm{~g}$ twice daily for 4 weeks. The OF-IN supplementation resulted in a significant decrease in Ruminococcus gnavus and a significant increase in the number of Bifidobacterium longum. Moreover, in the subgroup of patients with active CD there was a positive correlation between improvement in disease activity and an increase in the number of Bifidobacterium longum. The authors demonstrated that the CD-associated dysbiosis can be modified by OF-IN supplementation in patients with inactive and mild to moderately active CD [34]. A prospective, randomised, placebo-controlled pilot trial was conducted in a group of 19 patients with a relapse of mild to moderate activity of UC. They were on the same therapy with mesalamine (3 g per day) and they were randomly allocated to receive OF-IN (12 g per day) or placebo (12 g per day) for 2 weeks. OF-IN was well tolerated, and the dyspeptic symptoms scale decreased significantly with active treatment but not with placebo. At day 7 of intervention there was observed an early significant reduction of faecal calprotectin concentrations in the study group but not in the placebo group, so the anti-inflammatory effect of OF-IN was demonstrated.

The safety and efficacy of GBF supplementation has also been studied in some clinical trials in patients with UC. In a pilot study, 10 patients with mild to moderate active UC, who had been unresponsive to or intolerant of standard treatment, received $30 \mathrm{~g}$ GBF (three times daily) for 4 weeks. After 4 weeks of GBF supplementation there was a significant improvement in clinical activity and endoscopic index scores. Furthermore, a significant increase in stool butyrate concentrations was observed [35]. In another study, 59 patients with UC in remission, assessed according to Rachmilewitz's clinical activity index (CAI), were divided into a control group ( $n=37)$, which received standard treatment (glucocorticosteroids), and into a study group $(n=22)$, which received standard treatment plus $20 \mathrm{~g}$ GBF per day. Checkpoints were determined after 3, 6, and 12 months from the start of the intervention. Significantly lower CAI values and a lower percentage of relapses were observed in the study group, compared with the control group. The researchers concluded that GBF supplementation appeared to be safe and effective. It resulted in glucocorticosteroid dose reduction and it prolonged remission in patients with UC [36]. Faghfoori et al. [37] conducted a pilot study assessing the effect of GBF supplementation on serum CRP levels and frequency of clinical signs (number of diarrhoea episodes, degree of visible blood in stools, degree of abdominal pain or cramping, nausea, vomiting, and anorexia) in 46 patients with UC in remission. Patients were randomly assigned to the control group $(n=23)$ on standard therapy and study group $(n=23)$ on standard therapy plus GBF supplementation (30 g per day, three times in a day). At 2 months there was a significant decrease in mean serum CRP levels ( $p=0.017$ ) in the GBF group. There was a statistically significant reduction only in the frequency of abdominal pain and cramping ( $p=0.016)$; however, a decrease in the frequency of all clinical signs 
in the study group was observed, compared to the control group.

The authors of all of the presented GBF trails reported that there were no side effects related to GBF supplementation.

Hallert et al. [38] conducted a pilot study that evaluated the safety and efficacy of a high-fibre diet in 22 patients with UC in remission (median time from last relapse, one year before the intervention). The patients were recommended to add $60 \mathrm{~g}$ of oat bran per day (corresponding to $20 \mathrm{~g}$ dietary fibre) to the diet for 3 months. The oat bran supplementation resulted in a statistically significant increase in the faecal SCFA concentration, in particular butyrate. None of the patients in the study group reported gastrointestinal complaints and there were no colitis relapses. The trial demonstrated a good tolerance of oat bran intervention and its efficacy in increasing faecal SCFA concentrations in patients with UC who were in remission period.

\section{Conclusions}

Existing IBD guidelines from the Academy of Nutrition and Dietetics (formerly the American Dietetic Association), World Gastroenterology Organisation, and the Crohn's \& Colitis Foundation of America [10], regarding fibre intake, are very cautious: they all agree that dietary fibre consumption should not be limited in IBD patients in remission (except patients with strictures of the bowels).

Based on studies summarised in the article, dietary fibre shows significant clinical benefits in patients with IBD. Supplementation of some types of dietary fibre can help to maintain remission and reduce lesions of the intestinal mucosa during the course of the disease. The described effects are primarily associated with increased luminal production of SCFA after administering dietary fibre. SCFA have immunomodulatory properties, they accelerate healing and regeneration processes of the intestinal epithelium, and they lower colonic $\mathrm{pH}$ thereby stimulating growth of the beneficial microflora and inhibiting growth of the pathogens. Undoubtedly, more well-planned studies are need to establish whether dietary fibre plays an important role in IBD therapy or not.

\section{Conflict of interest}

The authors declare no conflict of interest.

\section{References}

1. Mamula P, Telega GW, Markowitz JE, et al. Inflammatory bowe disease in children 5 years of age and younger. Am J Gastroenterol 2002; 97: 2005-10.
2. Heyman MB, Kirschner BS, Gold BD, et al. Children with early-onset inflammatory bowel disease (IBD): analysis of a pediatric IBD consortium registry. J Pediatr 2005; 146: 35-40.

3. Khor B, Gardet A, Xavier RJ. Genetics and pathogenesis of inflammatory bowel disease. Nature 2011; 474: 307-17.

4. Xavier RJ, Podolsky DK. Unravelling the pathogenesis of inflammatory bowel disease. Nature 2007; 448: 427-34.

5. Radwan P, Radwan-Kwiatek K, Skrzydło-Radomańska B. The role of enteric microflora in inflammatory bowel disease. Prz Gastroenterol 2009; 4: 1-6.

6. Hou JK, Abraham B, El-Serag H. Dietary intake and risk of developing inflammatory bowel disease: a systematic review of the literature. Am J Gastroenterol 2011; 106: 563-73.

7. Burkitt DP. Epidemiology of large bowel disease: the role of fiber. Proc Nutr Soc 1973; 32: 145-9.

8. Heaton KW, Thornton JR, Emmett PM. Treatment of Crohn's disease with an unrefined-carbohydrate, fibre-rich diet. $\mathrm{Br}$ Med J 1979; 2: 764-6.

9. Zallot C, Quilliot D, Chevaux JB, et al. Dietary beliefs and behavior among inflammatory bowel disease patients. Inflamm Bowel Dis 2013; 19: 66-72.

10. Brown AC, Rampertab SD, Mullin GE. Existing dietary guidelines for Crohn's disease and ulcerative colitis. Expert Rev Gastroenterol Hepatol 2011; 5: 411-25.

11. ACC Dietary Fiber Definition Committee. Definition of dietary fiber: Report of the dietary fiber definition committee to the board of Directors of the American Association of Cereal Chemists. Cereal Food World 2001; 46: 112-26.

12. Codex Alimentarius Commission; Food and Agriculture Organization; World Health Organization. Report of the $30^{\text {th }}$ session of the Codex Committee on nutrition and foods for special dietary uses. ALINORM 09/32/26 2009 [cited 2014 Nov 30]. Available from: www.codexalimentarius.org.

13. Jones JM. CODEX-aligned dietary fiber definitions help to bridge the 'fiber gap'. Nutr J 2014; 13: 34.

14. FAO/WHO (Food and Agriculture Organization/World Health Organization), 1998. Carbohydrates in human nutrition. Report of a Joint FAO/WHO expert consultation. FAO Food and Nutrition Paper - 66, Rome.

15. Rosin PM, Lajolo FM, Menezes EW. Measurement and characterization of dietary starches. J Food Compos Anal 2002; 15: 367-77.

16. Champ MM. Physiological aspects of resistant starch and in vivo measurements. J AOAC Int 2004; 87: 749-55.

17. Topping DL, Clifton PM. Short-chain fatty acids and human colonic function: roles of resistant starch and nonstarch polysaccharides. Physiol Rev 2001; 81: 1031-64.

18. Schakel SF, Pettit J, Himes JH. Appendix - Table A.1. Dietary fiber values for common foods. In: CRC Handbook of dietary fiber in human nutrition. $3^{\text {rd }}$ ed. Spiller G (eds.). CRC Press LLC 2001; 615-41.

19. Institute of Medicine. Food and Nutrition Board. Dietary, functional and total fiber. In: Dietary reference intakes: energy, carbohydrates, fiber, fat, fatty acids, cholesterol, protein and amino acids. National Academies Press, Washington (DC) 2005; 339-421.

20. Rodríguez-Cabezas M, Gálvez J, Lorente MD, et al. Dietary fiber down-regulates colonic tumor necrosis factor alpha and nitric 
oxide production in trinitrobenzenesulfonic acid-induced colitic rats. J Nutr 2002; 132: 3263-71.

21. Rodríguez-Cabezas M, Gálvez J, Camuesco D. Intestinal anti-inflammatory activity of dietary fiber (Plantago ovata seeds) in HLA-B27 transgenic rats. Clin Nutr 2003; 22: 463-71.

22. Videla S, Vilaseca J, Antolín M, et al. Dietary inulin improves distal colitis induced by dextran sodium sulfate in the rat. Am J Gastroenterol 2001; 96: 1486-93.

23. Hoentjen F, Welling GW, Harmsen HJ, et al. Reduction of colitis by prebiotics in HLA-B27 transgenic rats is associated with microflora changes and immunomodulation. Inflamm Bowel Dis 2005; 11: 977-85.

24. Lara-Villoslada F, de Haro O, Camuesco D, et al. Short-chain fructooligosaccharides, in spite of being fermented in the upper part of the large intestine, have anti-inflammatory activity in the TNBS model of colitis. Eur J Nutr 2006; 45: 418-25.

25. Hino S, Ito $\mathrm{H}$, Bito $\mathrm{H}$, et al. Ameliorating effects of short-chain inulin-like fructans on the healing stage of trinitrobenzene sulfonic acid-induced colitis in rats. Biosci Biotechnol Biochem 2011; 75: 2169-74.

26. Koleva PT, Valcheva RS, Sun X, et al. Inulin and fructo-oligosaccharides have divergent effects on colitis and commensal microbiota in HLA-B27 transgenic rats. Br J Nutr 2012; 108: 1633-43.

27. Moreau NM, Martin LJ, Toquet CS, et al. Restoration of the integrity of rat caeco-colonic mucosa by resistant starch, but not by fructo-oligosaccharides, in dextran sulfate sodium-induced experimental colitis. Br J Nutr 2003; 90: 75-85

28. Kanauchi O, Nakamura T, Agata K, et al. Effects of germinated barley foodstuff on dextran sulfate sodium-induced colitis in rats. J Gastroenterol 1998; 33: 179-88.

29. Araki Y, Kanauchi O, Sugihara H, et al. Germinated barley foodstuff suppresses dextran sulfate experimental colitis in rats: the role of mast cells. Int J Mol Med 2007; 19: 257-62.

30. Davies PS, Rhodes J. Maintenance of remission in ulcerative colitis with sulphasalazine or a high-fibre diet: a clinical trial. Br Med J 1978; 1: 1524-5.

31. Hallert C, Kaldma M, Petersson BG. Ispaghula husk may relieve gastrointestinal symptoms in ulcerative colitis in remission. Scand J Gastroenterol 1991; 26: 747-50.

32. Fernández-Bañares F, Hinojosa J, Sánchez-Lombraña JL, et al. Randomized clinical trial of Plantago ovata seeds (dietary fiber) as compared with mesalamine in maintaining remission in ulcerative colitis. Spanish Group for the Study of Crohn's Disease and Ulcerative Colitis (GETECCU). Am J Gastroenterol 1999; 94: 427-33.

33. Welters CF, Heineman E, Thunnissen FB, et al. Effect of dietary inulin supplementation on inflammation of pouch mucosa in patients with an ileal pouch-anal anastomosis. Dis Colon Rectum 2002; 45: 621-7.

34. Joossens M, De Preter V, Ballet V, et al. Effect of oligofructose-enriched inulin (OF-IN) on bacterial composition and disease activity of patients with Crohn's disease: results from a double-blinded randomised controlled trial. Gut 2012; 61: 958.

35. Mitsuyama K, Saiki T, Kanauchi O, et al. Treatment of ulcerative colitis with germinated barley foodstuff feeding: a pilot study. Aliment Pharmacol Ther 1998; 12: 1225-30.

36. Hanai H, Kanauchi O, Mitsuyama K, et al. Germinated barley foodstuff prolongs remission in patients with ulcerative colitis. Int J Mol Med 2004; 13: 643-7.
37. Faghfoori Z, Shakerhosseini R, Navai L, et al. Effects of an oral supplementation of germinated barley foodstuff on serum CRP level and clinical signs in patients with ulcerative colitis. Health Promot Perspect 2014; 4: 116-21.

38. Hallert C, Björck I, Nyman M, et al. Increasing fecal butyrate in ulcerative colitis patients by diet: controlled pilot study. Inflamm Bowel Dis 2003; 9: 116-21.

Received: 7.01.2015

Accepted: 24.02.2015 